\title{
The state of the Universe
}

\section{A bold attempt to make sense of relativity, quantum theory and cosmology.}

\section{The Road to Reality: A Complete Guide to the Laws of the Universe by Roger Penrose}

Jonathan Cape: 2004. 1,094 pp. $£ 30$

\section{Jeffrey Forshaw}

"The most important and ambitious work of science for a generation." That's the claim from the publishers of Roger Penrose's latest book. The claim is vastly overblown. Certainly Penrose has written a remarkable book: it introduces many of the topics that lie at the cutting edge of research into the fundamental nature of space, time and matter. Although the book aims at a complete survey of modern particle physics and cosmology, its principal concern is to address the fundamental tension between the two pillars of twentieth-century physics: Einstein's general theory of relativity and quantum theory. This is a fascinating tension and one that Penrose tries to communicate in a quite uncompromising fashion.

Although advertised as popular science, this book will be far from accessible to most non-experts. I suspect that there has never been such a bold attempt to communicate ideas of such mathematical complexity to a general audience. It is Penrose's hope that non-experts will be able to go with the flow and get a taste of the excitement of the field without following the details. Unfortunately, the book is crammed full of details and is so utterly uncompromising that it will probably leave even the most enthusiastic of his nonexpert readership exhausted. Even those with a $\mathrm{PhD}$ in mathematics or physics are likely to find it very hard going.

Having said that, the book is teeming with delights. Penrose's ability to present complex ideas in a logical, coherent manner, often using geometry, reflect a deep understanding that will seriously engage a more expert reader. There are even exercises, graded "very straightforward", "needs a bit of thought" and "not to be undertaken lightly", which the reader is encouraged to attempt. I certainly found the classification of this final category quite appropriate.

The opening chapter, on the roots of science and the notion of mathematical truth, marvels over the ability of mathematics to explain so much of the behaviour of the world with such incredible precision. Some scientists may balk at questions of ontology, preferring to banish them to the realm of metaphysics, but this is not Penrose's way. In his subsequent assessment of the measurement problem in quantum mechanics, he argues forcefully for the need for a credible ontology. The book ends with reflections on

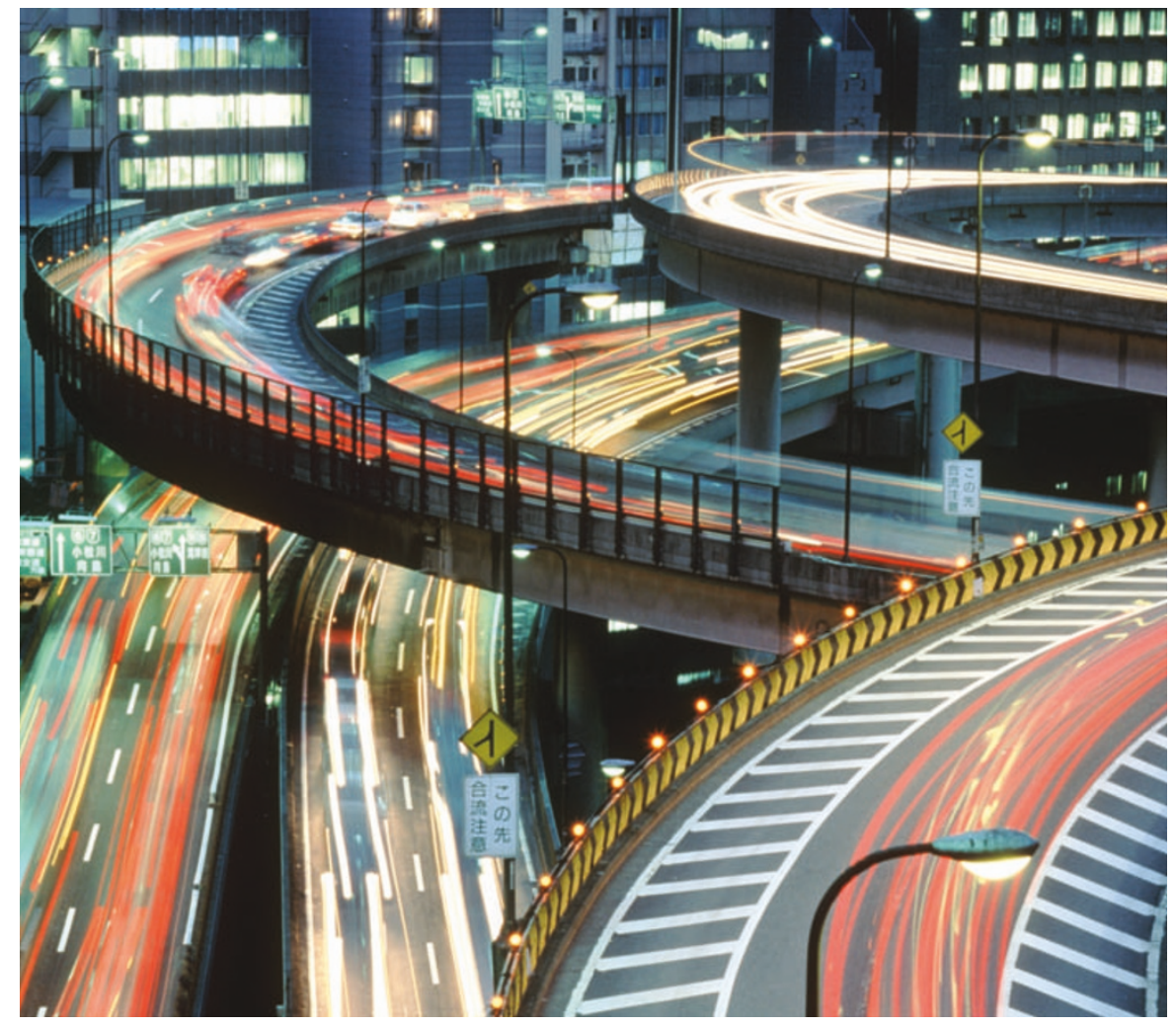

Up the junction? Despite progress in many directions, we still haven't found the one "road to reality".

the future, critically assessing the way in which physicists progress in their quest for an ever deeper comprehension of reality, and offering some personal views on the sociology of contemporary research. In between there are 32 chapters of serious mathematical physics with little room for philosophical reflections.

So serious is Penrose in his desire to present the science in a worthy fashion that one has to wait patiently (or struggle valiantly) until chapter 17 for the physics. The mathematics of the first part of the book is a joy to read. Not all of it is needed for the later chapters, and the selection and presentation of material clearly reflects Penrose's love of the mathematical structures that underpin the physics.

Penrose starts from a clean slate, encouraging the reader to question the dominant role of real numbers, or the validity of euclidean geometry, in describing the world around us. Much time is devoted to complex numbers and their "magic". In typical fashion, Penrose does not shy from introducing Riemann surfaces, holomorphicity and hyperfunctions. Hot on the heels of these come quaternions, leading naturally to a discussion of Clifford and Grassman algebras. Chapters on differential geometry and symmetry conclude the presentation of the necessary mathematics. The section ends with a chapter - not strictly relevant but entirely captivating - on the notion of infinity, covering Cantor's work on cardinal numbers and Gödel's incompleteness theorem.

Then the physics starts, with a chapter on space-time. The earlier chapter on differential geometry has paved the way and the reader is ready to understand Galileo's principle of relativity as implying that "space is a bundle over time". Einstein's idea that space and time might constitute a unified whole is by now an entirely reasonable a priori possibility.

The following chapters introduce field theory and quantum theory. Much of the discussion on the measurement problem in quantum theory will be familiar to those who have read Penrose's earlier book The Emperor's New Mind. In The Road to Reality he lays out his contention that quantum theory, as currently formulated, must be incomplete, and this is a recurrent theme for the remainder of the book.

There is a rather cursory chapter on the standard model of particle physics, which rather confusingly precedes the chapter on quantum field theory and makes the error of stating that weak interactions couple only to left-handed particles. It is also stated that the only measured violation of charge 
parity, a symmetry that ultimately helps to account for the preponderance of matter over antimatter in the Universe, is in the study of neutral kaon mesons, a declaration that will not be popular with those working on certain experiments based in the United States and Japan.

The main thrust of the final part of the book is the exploration of physics in those often exotic places where both quantum mechanics and gravity play a role, such as at the birth of the Universe or in the vicinity of a black hole. The very existence of the second law of thermodynamics is used to argue that the Universe must have started out in an extraordinarily special configuration (something else that will be familiar to readers of The Emperor's New Mind), which Penrose takes as a hint that the quantum theory requires revision.

After a more detailed discussion of the measurement paradox, Penrose is ready to expound his belief that quantum state reduction is an objective process, arising as a consequence of the gravitational energy difference between the different space-time geometries possessed by quantum states in superposition. By now, Penrose is becoming increasingly partisan, although he is careful to announce when he is deviating from the "accepted wisdom". He is forceful in stating his objections to several mainstream ideas: inflationary cosmology, low-energy supersymmetry, the electroweak phase transition and string theory are all targets. Indeed, his presentation of string theory is essentially a technical critique, and I suspect it will be inaccessible to non-experts. At times his objections can lead to rather judgemental and acerbic statements: the leading string theorist, Ed Witten, is described at one point as a "tour guide".

Having been so critical, Penrose offers his thinking on the possible way forward for a consistent theory of quantum gravity. Unfortunately these two chapters are the most challenging of the whole book. Loop variables, spin networks and his own twistor theory are all presented, although he acknowledges that these ideas have not yet come close to matching up as a viable physical theory.

In sum, this book certainly doesn't live up to the publisher's hype. It is too technical to be accessible to a general audience and is focused on only one branch of modern science. However, it is carefully crafted and rich in deep insight. Penrose's own hand drawings help to remind the reader of the very personal account provided in its pages. Penrose, leading by example, clearly intends that this book should encourage scientists to dare to be original in their quest: in that, he may well have succeeded.

Jeffrey Forshaw is in the Department of Physics and Astronomy, University of Manchester,

Oxford Road, Manchester M13 9PL, UK.

\section{Field guides and phylogenies}

\section{Flowers of Ethiopia and Eritrea: \\ Aloes and Other Lilies \\ by Sebsebe Demissew, Inger Nordal \& \\ Odd E. Stabbetorp \\ Shama Books, Addis Ababa, Ethiopia: 2004. \\ 227 pp. US $\$ 15$}

e-mail: shamabooks@telecom.net.et

\section{Sandra Knapp}

Taxonomy has been much in the news in the past year or so. Everybody seems to want more of it, and in a slightly different way than it is currently done. Perhaps taxonomy should be done solely on the web, or by using a set of DNA barcodes - opinions differ. But just what is taxonomy and why is it suddenly in demand? I contend that taxonomy (or systematics, as some people prefer to call it) is composed of three interlocking spheres of scientific endeavour: phylogeny, description and identification. This book is a sterling example of how all three aspects of taxonomy can come together to produce something of lasting value to a variety of end-users.

The book is a colour guide to the identification of the charismatic flowers that characterize dry habitats such as Ethiopia 'lilies' in the broadest sense. Identification is aided by easy-to-use keys, simple descriptions and lovely photographic plates, which make this a nice book for just exploring the amazing diversity of these plants, quite apart from its obvious use in the field. But the authors have done more than just produce an identification guide: they have set the lilies of the region in their phylogenetic context, and the book's introduction shows just how important it is to link those three areas of taxonomic study.

The phylogeny of the monocots (such as lilies, grasses and orchids) has undergone radical change and restructuring with the use of DNA sequence data. The number of families recognized and their relationships to one another have changed considerably over the past decade (see Bot. J. Linn. Soc. 141, 399-436; 2003). Rather than brushing all this under the carpet, the authors clearly and concisely explain why phylogeny matters. Then, through the use of the field-guide format, they show how such rearrangements make sense when looking at the plants themselves. They are also brave enough to admit what all taxonomists know: that the classification of such groups, where new data are emerging, is still in flux.

Phylogenies and field guides are both of obvious use, but both require a solid base of descriptive taxonomy. This book, and to a certain extent the phylogeny to which it adheres, rests on the descriptive work done on the region's flora. Major projects documenting national floras (and faunas) have uncovered new species and provided the observations and taxonomic decisions that are needed for both phylogeny and field identification.

Books such as this, with its numerous illustrations and accessible style, inspire others to study organisms, helping the accumulation of information about uses and, to my mind more importantly, conservation status. How on earth can we conserve biodiversity unless we know something about it? This book is an example of how to do it.

Sandra Knapp is in the Department of Botany, The Natural History Museum, Cromwell Road, London SW7 5BD, UK.

\section{Sculpture}

\section{Opening time}

On a summer evening several years ago, Eduardo Catalano, emeritus professor of architecture at the Massachusetts Institute of Technology, watched a flower as it was starting to close its petals. Suddenly, he realized what the movable structure he had so long wanted to create should look like.

That moment of inspiration resulted in 13 months of construction work. The outcome was Floralis Genérica, a stunning, gigantic flower (right) made of an aluminium alloy covered in stainless steel, intended to represent all flowers. It is 20 metres high and weighs 18 tons. Each of its six petals is 13 metres long and 7 metres wide, and they are mounted on a conical structure. A computer-controlled hydraulic mechanism opens the flower at sunrise and closes it at dusk. Red light is projected on the inner surface of the petals when the flower closes.

Born in Argentina, Catalano donated the

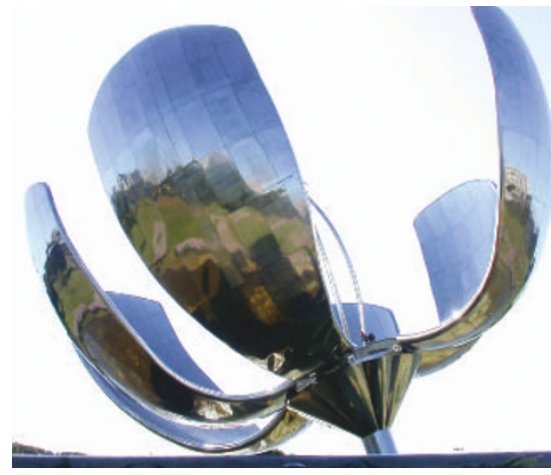

flower to the city of Buenos Aires, where it was inaugurated in April 2002. Floralis Genérica has found its home in a large pond in the heart of the city. The flower is kept open round the clock on the first day of spring and on some public holidays. And whenever there is a new Moon such as today. 